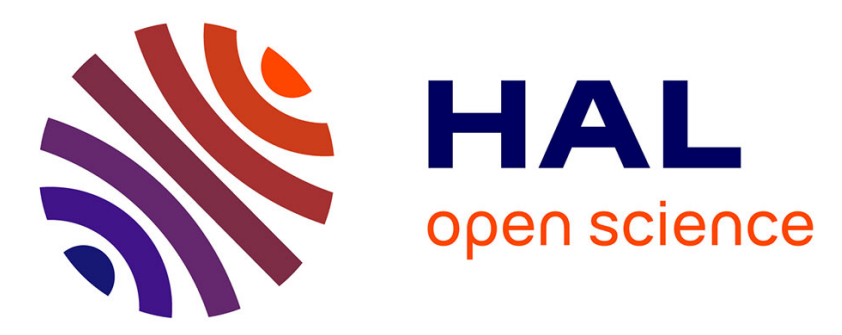

\title{
Validation of a 2D multispectral camera: application to dermatology/cosmetology on a population covering five skin phototypes
}

Romuald Jolivot, Hermawan Nugroho, Pierre Vabres, Ahmad Fadzil, Franck S. Marzani

\section{To cite this version:}

Romuald Jolivot, Hermawan Nugroho, Pierre Vabres, Ahmad Fadzil, Franck S. Marzani. Validation of a 2D multispectral camera: application to dermatology/cosmetology on a population covering five skin phototypes. European Conference on Biomedical Optics, SPIE, May 2011, Munich, Germany. pp.Proc. SPIE 8087, 808729, 10.1117/12.889607 . hal-00614126

\section{HAL Id: hal-00614126 \\ https://hal.science/hal-00614126}

Submitted on 9 Aug 2011

HAL is a multi-disciplinary open access archive for the deposit and dissemination of scientific research documents, whether they are published or not. The documents may come from teaching and research institutions in France or abroad, or from public or private research centers.
L'archive ouverte pluridisciplinaire HAL, est destinée au dépôt et à la diffusion de documents scientifiques de niveau recherche, publiés ou non, émanant des établissements d'enseignement et de recherche français ou étrangers, des laboratoires publics ou privés. 


\title{
Validation of a 2D multispectral camera: application to dermatology/cosmetology on a population covering five skin phototypes
}

\author{
Romuald Jolivot $^{a}$, Hermawan Nugroho ${ }^{b}$, Pierre Vabres ${ }^{a c}$, M. H. Ahmad Fadzil ${ }^{b}$ and Franck \\ Marzani $^{a}$ \\ ${ }^{a}$ Laboratoire Le2i, UMR CNRS 5158, Université de Bourgogne, BP 47870, 21078 Dijon Cedex, \\ France; \\ ${ }^{b}$ Universiti Teknologi Petronas, Bandar Seri Iskandar, 31750 Tronoh, Malaysia; \\ ${ }^{c}$ Dept. of Dermatology, University Hospital, BP 77908, 21079 Dijon Cedex, France.
}

\begin{abstract}
This paper presents the validation of a new multispectral camera specifically developed for dermatological application based on healthy participants from five different Skin PhotoTypes (SPT). The multispectral system provides images of the skin reflectance at different spectral bands, coupled with a neural network-based algorithm that reconstructs a hyperspectral cube of cutaneous data from a multispectral image. The flexibility of neural network based algorithm allows reconstruction at different wave ranges. The hyperspectral cube provides both high spectral and spatial information. The study population involves 150 healthy participants. The participants are classified based on their skin phototype according to the Fitzpatrick Scale and population covers five of the six types. The acquisition of a participant is performed at three body locations: two skin areas exposed to the sun (hand, face) and one area non exposed to the sun (lower back) and each is reconstructed at 3 different wave ranges. The validation is performed by comparing data acquired from a commercial spectrophotometer with the reconstructed spectrum obtained from averaging the hyperspectral cube. The comparison is calculated between 430 to $740 \mathrm{~nm}$ due to the limit of the spectrophotometer used. The results reveal that the multispectral camera is able to reconstruct hyperspectral cube with a goodness of fit coefficient superior to 0,997 for the average of all SPT for each location. The study reveals that the multispectral camera provides accurate reconstruction of hyperspectral cube which can be used for analysis of skin reflectance spectrum.
\end{abstract}

Keywords: Skin imaging system, multispectral image, hyperspectral cube, spectral reflectance, spectral reconstruction, validation

\section{INTRODUCTION}

Skin lesion evaluations are mainly based on naked eyes/RGB camera analysis. However such analysis are limited due to poor discrimination of the human eye. It is linked to the fact that colour sensed by the human eye is a combination of three different bands called trichromatic model. Intensive researches in the non-invasive skin imaging area bring new imaging techniques that work as an adjunct help system and for some even surpass the human eye. The motivation is to go beyond the limited red, green, blue color imaging as it reflects the human light perception. The human eyes and RGB cameras are affected by a phenomenon called metamerism: two colour samples match under the same illuminant but may appear totally different when using a different illuminant; similarly, for skin lesions observed under different condition, the analysis made by a dermatologist can be affected by this phenomenon. Imaging techniques offer capacity to get extra information about the skin to the dermatologist for diagnosis assessment. Those efforts try to bring objective measurement of skin lesions. It can be done either by a spectroscopic measurement ${ }^{1,2}$ or by imaging techniques ${ }^{3,4}$. Combining advantages of both spectrophotometer (spectral resolution) and digital camera (spatial resolution), MultiSpectral Imaging (MSI) systems overcome their respective limitations (lack of spatial variation and lack of spectral information).

Further author information: (Send correspondence to Romuald Jolivot)

: E-mail: romuald.jolivot@u-bourgogne.fr, Telephone: +33 (0)3 80395934 
The aim of this work is to prove the accurate reconstruction of hyperspectral cube of cutaneous data from multispectral images. In order to validate this, a comparison study between a commercial spectrophotometer and the ASCLEPIOS $^{5}$ camera developed in our team has been performed on a study population covering different skin tones.

\section{MATERIALS}

The acquisition device used in the study is a multispectral camera covering visible and near infra red spectrum, called ASCLEPIOS (see figure 1). It is an extended version of a previous multispectral camera limited to the visible $^{6}$. The MSI is composed of a Xenon light source covering wavelength range from $340 \mathrm{~nm}$ to infrared light. The light compartment houses a rotating wheel holding a set of interference filters which gradually rotates to position each filter in front of the light source. The spectral band of the set of ten interference filters covers from $430 \mathrm{~nm}$ to $970 \mathrm{~nm}$. An opaque custom built hand-held device encloses a liquid light guide, which transmits the illumination, a camera and a remote wireless trigger. The camera consists of a monochromatic CMOS digital camera with spectral response from 370 to $1100 \mathrm{~nm}$ and resolution of $1312 \times 1082$ pixels. The hand-held device, in contact with the skin, keeps a constant focus distance between the camera and the skin area. A magnifying lens is mounted in front of the camera which provides an useful area of $32 x 38 \mathrm{~mm}$ size with a depth of field of $5 \mathrm{~mm}$, yielding a spatial resolution of 33 pixels. $\mathrm{mm}^{-1}$. The acquisition is performed in less that 2 seconds by illuminating the skin at different wavebands after the automatic rotation of the wheel for each filter. The system acquires one multispectral image composed of ten monobands.

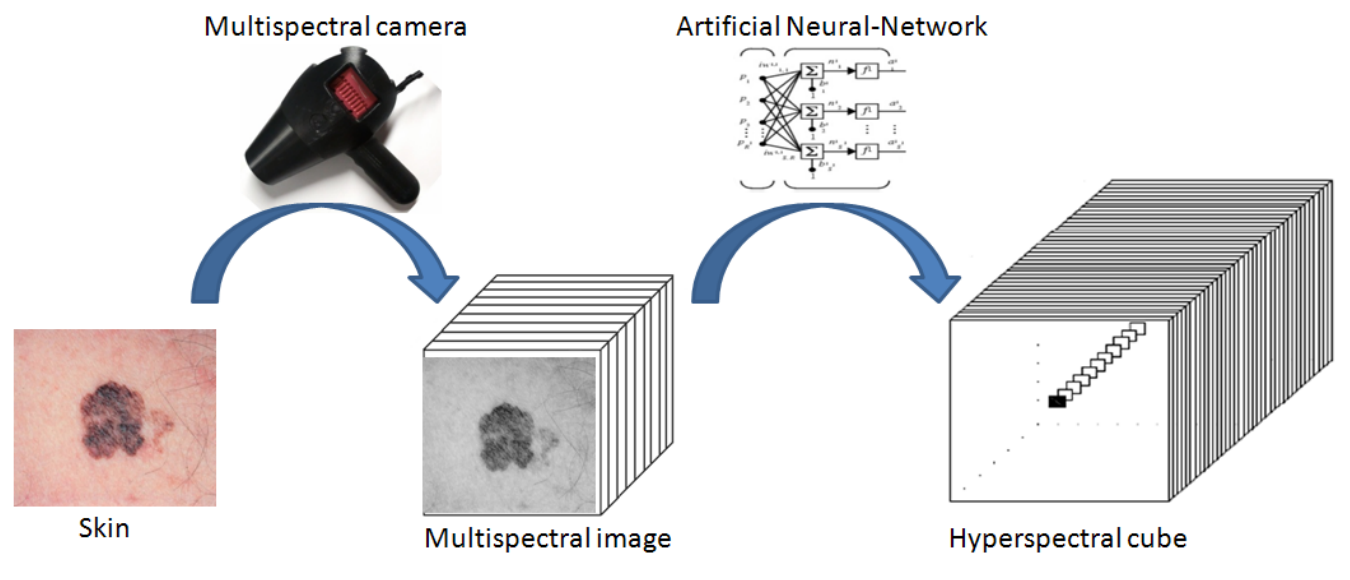

Figure 1. Asclepios system

The originality of the ASCLEPIOS system is its capacity to reconstruct a Hyperspectral Cube from a multispectral image. This reconstruction is performed by an Artificial Neural Network-based (ANN) algorithm using hetero-associative memory. ${ }^{7}$ The reconstruction aims to retrieve skin reflectance spectra in each pixel from the camera signal. The reconstructed hyperspectral cube of cutaneous data provides a 3-dimensional volume $(\mathrm{x}, \mathrm{y}, \mathrm{z})$ where $\mathrm{x}$ and $\mathrm{y}$ are for spatial dimensions and $\mathrm{z}$ for spectral one. The ANN is divided into two parts, first a learning process based on GretagMacBeth ColorChecker and then a reconstruction process. Due to the use of hetero-associative memory, the algorithm is able to reconstruct hyperspectral cube with different wave ranges within the system capability (430 to $970 \mathrm{~nm}$ ) and step up to $1 \mathrm{~nm}$.

\section{METHODS}

The goal of hyperspectral cube reconstruction is to retrieve skin information from the reflectance spectrum leading to an increase of the amount of information available to the dermatologist for diagnosis purpose. To validate the accuracy of the reconstruction of hyperspectral cube of cutaneous data, a study on healthy participants from different skin tones has been performed. The classification of the different skin tones is based on the Fitzpatrick Skin PhotoType ${ }^{8}$ (SPT) which classify a person's complexion and tolerance to sunlight. According 


\begin{tabular}{|l|c|c|c|c|c|}
\hline SPT Type & SPT II & SPT III & SPT IV & SPT V & SPT VI \\
\hline $\mathrm{Nb}$ & 18 & 21 & 56 & 41 & 14 \\
\hline
\end{tabular}

to the Fitzpatrick scale, human skin can be divided into six categories, SPT I for very white skin (never tans and always burns) to SPT VI for black skin (never burns). The different SPT aims to show accurate reconstruction for a wide range of spectrum. Three acquisitions (by a commercial spectrophotometer CS 2600d and by the multispectral camera) were collected from each participant of the study: two data were acquired from facultative skin colour and one from constitutive skin colour. Facultative skin colour is skin area affected by the environment (sun, hormones) and it impacts the original appearance of the skin colour over time whereas constitutive skin colour is determined by genetics. For facultative colour, one sample from the back of the hand and one sample from the face are acquired. For constitutive skin colour, one sample from the lower back is acquired. The purpose of different body locations is to record a difference of skin chromophore composition between these samples. This is due to the fact that the face and the back of the hand are supposed to be more exposed to the sun than the lower back. Melanin production is linked to sun exposure (UV), these two body locations are expected to have higher melanin concentration and therefore they should have a different skin reflectance spectrum. The aim is to observe a difference within each participant between exposed (facultative skin colour) and non-exposed (constitutive skin colour) area.

In this study, using the wave-range flexibility offered by the ANN, three reconstruction configurations are selected, one reconstruction from 430 to $780 \mathrm{~nm}$, another one from 430 to $850 \mathrm{~nm}$ and a third one from 430 to $950 \mathrm{~nm}$ with a step of $10 \mathrm{~nm}$. The objective of testing different wave-range is to select the best configuration for accurate reconstruction for visible light range.

The study involves 150 recruited healthy (with no known skin disease) participants of different skin tones. The acquisition was conducted at University Teknologi PETRONAS (Malaysia) on international and local undergraduate and postgraduate students. The population covers five out of the six SPT groups, no participant of type I were available for the study. The distribution within the different SPT is shown in table 1. The average age of the study population is 22.6 years with a standard deviation of 4.2 years.

To evaluate the performance of the reconstruction of hyperspectral cube, three different metric scales are employed, the Root Mean Squared Error (RMSE), the Goodness of Fit Coefficient (GFC) and the Reconstruction Percentage (RecP). These metric scale use the spectrophotometer data as reference.

The RMSE calculates the mean of euclidean distance values between two spectra. Reconstruction is good when RMSE is close to zero. RMSE is calculated using the following formula:

$$
R M S E=\frac{1}{N} \sum_{j=1}^{N}\left\|R_{m}\left(\lambda_{j}\right)-R_{r}\left(\lambda_{j}\right)\right\|^{2}
$$

where $R_{m}\left(\lambda_{j}\right)$ is the spectrum value measured with the spectrophotometer at wavelength $\lambda_{j}$ and $R_{r}\left(\lambda_{j}\right)$ is the reconstructed spectra value at wavelength $\lambda_{j}$ and $N$ the number of samples.

The GFC is based on the Schwartz inequality and is calculated using the following formula:

$$
G F C=\frac{\left|\sum_{j} R_{m}\left(\lambda_{j}\right) R_{r}\left(\lambda_{j}\right)\right|}{\left(\sum_{j}\left[R_{m}\left(\lambda_{j}\right)\right]^{2}\right)^{1 / 2}\left(\sum_{j}\left[R_{r}\left(\lambda_{j}\right)\right]^{2}\right)^{1 / 2}} .
$$

This criterion has the advantage to be bounded between 0 and 1, providing an easy interpretation.

The RecP is a criterion which evaluates the distance between two spectra. It requires a higher precision for the spectrum part having low amplitude. A good reconstruction will be close to 100\%. It is defined by:

$$
R e c P=1-\frac{\sum_{j=1}^{N}\left\|R_{m}\left(\lambda_{j}\right)-R_{r}\left(\lambda_{j}\right)\right\|^{2}}{\sum_{j=1}^{N}\left\|R_{r}\left(\lambda_{j}\right)\right\|^{2}} .
$$




\section{RESULTS}

The data obtained from the spectrophotometer is a spectrum from $360 \mathrm{~nm}$ to $740 \mathrm{~nm}$ with a step of $10 \mathrm{~nm}$. The hyperspectral cube is reconstructed using the three configuration previously mentioned (430-780 nm, 430-850 $\mathrm{nm}, 430-950 \mathrm{~nm}$ ) all with a step of $10 \mathrm{~nm}$.

The results are divided into six groups (see table 2). The first five sets of rows concern the average of each SPT according to their locations and reconstruction wave-ranges. The last row of table 2 is the average of the all SPT data for each location and reconstruction wave-range.

The average of all the data reveal that the GFC, RMSE and RecP for the hand, face and lower back is higher for reconstruction between 430 to $780 \mathrm{~nm}$. It is also important to notice that the values of other reconstructions (430 to $850 \mathrm{~nm}$ and 430 to 950) are all superior to 0.9967 for the GFC. According to Hernandez et al.,${ }^{9}$ reconstruction with GFC higher than 0.99 is considered good.

The GFC result for each specific SPT group shows that reconstruction for SPT II and III appears to be better in the reconstruction range of 430 to 850 whereas reconstruction for SPT IV, V and VI obtains better results in the range of 430 to 780 . Similar conclusion arises for the PRec values and the RMSE.

\begin{tabular}{|c|c|c|c|c|c|c|c|c|c|}
\hline SPT II & Hand 780 & \begin{tabular}{|l|l} 
Hand 850 \\
\end{tabular} & Hand 950 & Face 780 & Face 850 & Face 950 & Back 780 & Back 850 & \begin{tabular}{|l|} 
Back 950 \\
\end{tabular} \\
\hline$\overline{\mathrm{GFC}}$ & 0,9977 & 0,9977 & 0,9970 & 0,9963 & 0,9964 & 0,9958 & $\begin{array}{l}0,9963 \\
\end{array}$ & 0,9965 & 0,9957 \\
\hline$\overline{\text { PRec }}$ & 0,9842 & 0,9846 & 0,9838 & 0,9776 & 0,9777 & 0,9694 & 0,9793 & 0,9809 & 0,9724 \\
\hline$\overline{\mathrm{RMSE}}$ & 18,87 & 18,30 & 18,91 & 32,23 & 32,11 & 44,42 & 35,15 & 32,67 & 47,93 \\
\hline$\overline{\overline{\text { SPT III }}}$ & 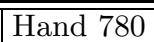 & Hand 850 & \begin{tabular}{|l|l} 
Hand 950 \\
\end{tabular} & Face 780 & \begin{tabular}{|l|} 
Face 850 \\
\end{tabular} & \begin{tabular}{l|l} 
Face 950 \\
\end{tabular} & Back 780 & Back 850 & \begin{tabular}{|l|} 
Back 950 \\
\end{tabular} \\
\hline GFC & 0,9980 & \begin{tabular}{|c|}
0,9980 \\
\end{tabular} & 0,9974 & 0,9972 & \begin{tabular}{|l|}
0,9973 \\
\end{tabular} & 0,9966 & 0,9974 & \begin{tabular}{|l}
0,9975 \\
\end{tabular} & \begin{tabular}{|c|}
0,9967 \\
\end{tabular} \\
\hline PRec & 0,9852 & 0,9856 & 0,9821 & 0,9797 & 0,9674 & 0,9677 & 0,9847 & 0,9848 & 0,9745 \\
\hline RMSE & 12,78 & 12,37 & 20,02 & 22,08 & 37,31 & 38,22 & 20,88 & 20,74 & 34,54 \\
\hline$\overline{\text { SPT IV }}$ & $\overline{\text { Hand } 780}$ & "Hand 850 & \begin{tabular}{|l|l} 
Hand 950 \\
\end{tabular} & \begin{tabular}{|l} 
Face 780 \\
\end{tabular} & Face 850 & \begin{tabular}{l|l} 
Face 950 \\
\end{tabular} & Back 780 & Back 850 & Back 950 \\
\hline GFC & 0,9976 & 0,9975 & \begin{tabular}{|c|}
0,9970 \\
\end{tabular} & $\begin{array}{c}0,9972 \\
\end{array}$ & \begin{tabular}{|c|}
0,9972 \\
\end{tabular} & 0,9966 & \begin{tabular}{|l|}
0,9975 \\
\end{tabular} & 0,9976 & \begin{tabular}{|c|}
0,9969 \\
\end{tabular} \\
\hline PRec & 0,9792 & 0,9788 & 0,9749 & 0,9802 & 0,9797 & 0,9727 & 0,9781 & 0,9775 & $\overline{0,9612}$ \\
\hline$\overline{\mathrm{RMSE}}$ & 16,30 & 16,67 & 17,55 & 20,84 & 21,32 & 27,74 & 21,06 & 21,53 & 37,15 \\
\hline$\overline{\overline{\text { SPT V V }}}$ & Hand 780 & Hand 850 & \begin{tabular}{|l} 
Hand 950 \\
\end{tabular} & Face 780 & \begin{tabular}{|l|} 
Face 850 \\
\end{tabular} & Face 950 & Back 780 & Back 850 & Back 950 \\
\hline$\overline{\mathrm{GFC}}$ & 0,9971 & 0,9968 & \begin{tabular}{|c|}
0,9963 \\
\end{tabular} & 0,9971 & \begin{tabular}{|c|}
0,9970 \\
\end{tabular} & 0,9965 & \begin{tabular}{|c|}
0,9980 \\
\end{tabular} & 0,9979 & \begin{tabular}{|c|}
0,9972 \\
\end{tabular} \\
\hline PRec & 0,9814 & 0,9793 & 0,9807 & 0,9836 & 0,9839 & 0,9790 & 0,9765 & 0,9767 & 0,9548 \\
\hline RMSE & 15,61 & 16,94 & 15,67 & 13,22 & 13,05 & 15,71 & 15,53 & 15,31 & 29,45 \\
\hline$\overline{\text { SPT VI }}$ & $\begin{array}{l}\text { Hand } 780 \\
\end{array}$ & Hand 850 & \begin{tabular}{|l|} 
Hand 950 \\
\end{tabular} & Face 780 & Face 850 & 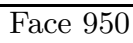 & Back 780 & Back 850 & Back 950 \\
\hline GFC & 0,9972 & 0,9961 & 0,9962 & 0,9977 & \begin{tabular}{|c|}
0,9969 \\
\end{tabular} & 0,9967 & 0,9975 & 0,9962 & \begin{tabular}{|c|}
0,9961 \\
\end{tabular} \\
\hline$\overline{\text { PRec }}$ & 0,9842 & 0,9797 & 0,9790 & 0,9860 & 0,9726 & 0,9775 & 0,9631 & 0,9691 & 0,9251 \\
\hline RMSE & 4,89 & 5,92 & 6,35 & 4,44 & 8,51 & 6,80 & 7,81 & 7,03 & 16,20 \\
\hline ALL SPT & Hand 780 & \begin{tabular}{|l|} 
Hand 850 \\
\end{tabular} & \begin{tabular}{|l|} 
Hand 950 \\
\end{tabular} & Face 780 & \begin{tabular}{|l|} 
Face 850 \\
\end{tabular} & Face 950 & Back 780 & Back 850 & \begin{tabular}{|l|} 
Back 950 \\
\end{tabular} \\
\hline GFC & 0,9975 & \begin{tabular}{|c|}
0,9973 \\
\end{tabular} & \begin{tabular}{|c|}
0,9968 \\
\end{tabular} & $\begin{array}{c}0,9971 \\
\end{array}$ & \begin{tabular}{|c|}
0,9970 \\
\end{tabular} & 0,9965 & 0,9975 & 0,9974 & \begin{tabular}{|c|}
0,9968 \\
\end{tabular} \\
\hline PRec & 0,9817 & 0,9807 & 0,9790 & 0,9813 & 0,9782 & 0,9738 & 0,9773 & 0,9778 & 0,9590 \\
\hline RMSE & 14,86 & 15,33 & 16,50 & 18,77 & 21,40 & 25,97 & 19,77 & 19,42 & 33,71 \\
\hline
\end{tabular}

Table 2. GFC, PRec and RMSE for each location and reconstruction range of the average data of each SPT and average of all SPT.

The results can be visually assessed with figure 2 and figure 3 which respectively show the average data per SPT acquired from the back of the hand for the spectrophotometer and Asclepios.

The shape of the curve from Asclepios is in good accordance with the curve obtained from the spectrophotometer.

Difference between constitutive and facultative skin colours is obtained by both system. Figures 4 and 5 shows respectively the average SPT III data acquired from the lower back (constitutive skin colour) and the back of the hand (facultative). The constitutive skin colour have higher spectral reflectance compare to the facultative one. 


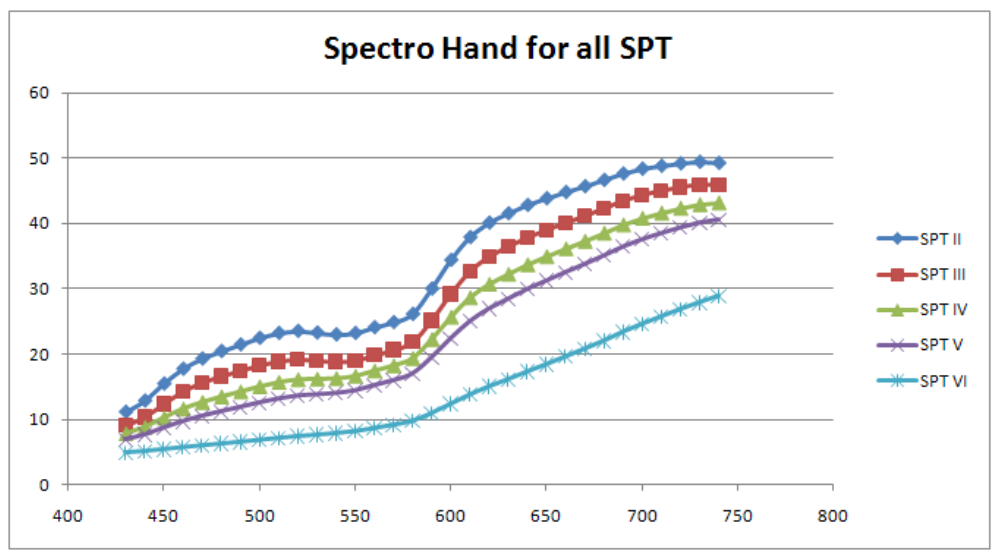

Figure 2. Spectrophotometer data from back of the hand.

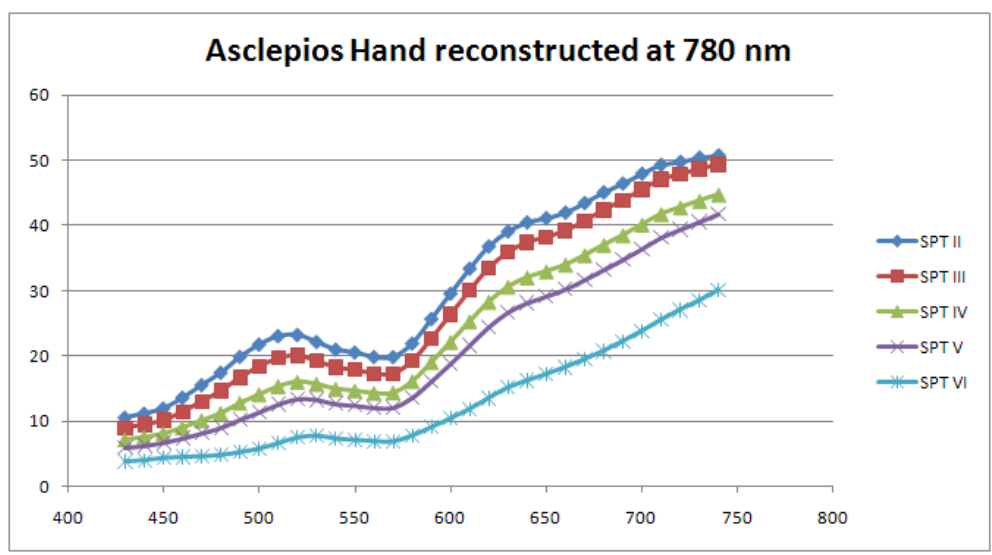

Figure 3. Asclepios data from back of the hand.

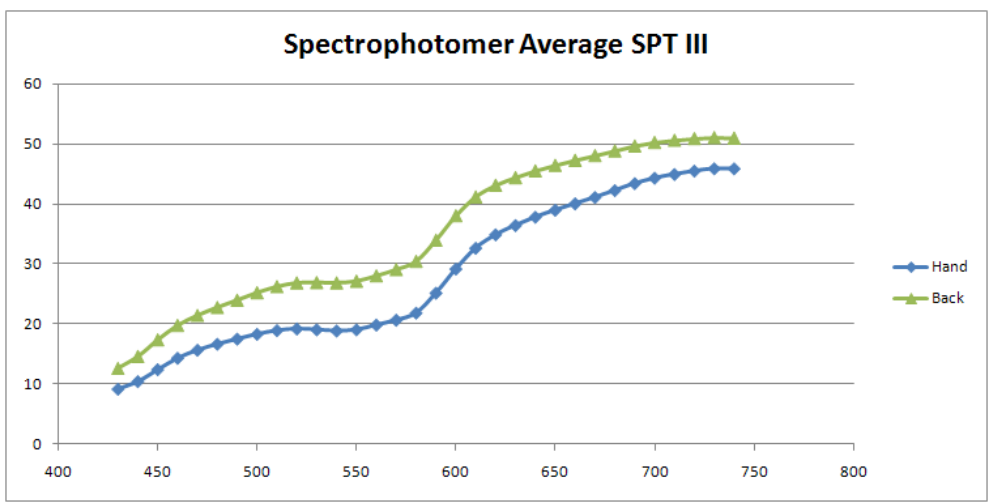

Figure 4. Average SPT III at two different locations acquired by CS2600d. 


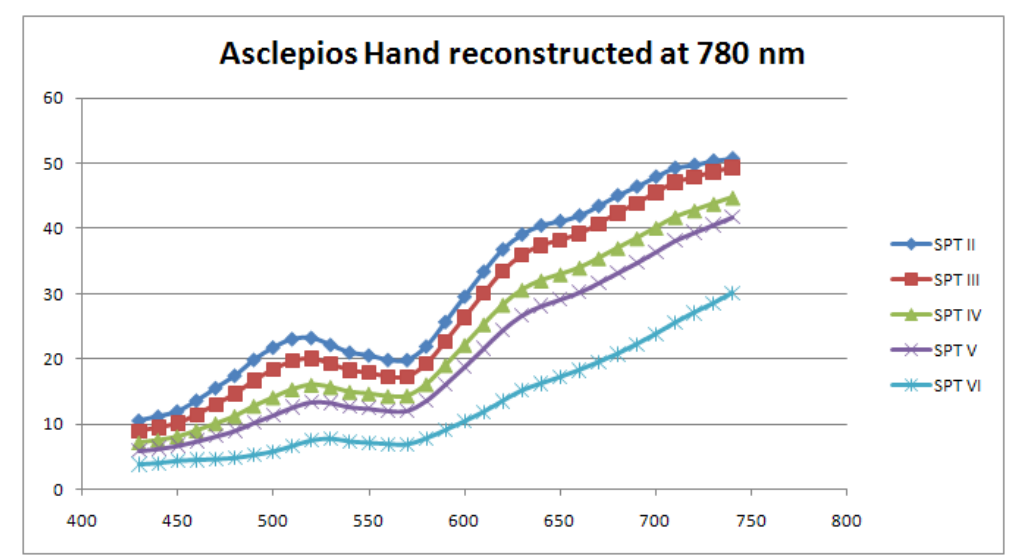

Figure 5. Average SPT III at two different locations acquired by Asclepios.

The difference of spectrum shape between area exposed to the sun against area non exposed to the sun can also be visually assessed. This revelation means that quantification of skin chromophores is possible with the two systems. However, with a spectrophotometer, several acquisitions are required whereas with Asclepios and its hyperspectral cube, only one acquisition is necessary.

Both numerical and visual analysis of the results reveal that the hyperspectral cube is accurately reconstruct.

\section{CONCLUSION}

This study validates the capacity of ASCLEPIOS system to accurately reconstruct hyperspectral cube of cutaneous data, which provides more information than conventional spectrophotometer, which provides only the average spectrum of an areas. Such hyperspectral cube provides both spectral and spatial information. The capacity of the system to discriminate area with different melanin composition, shows that within a same hyperspectral cube, the spectral information can be obtained offering a wide range of analysis to be performed (spectral analysis, morphological analysis) like source separation to extract within a single acquisition or follow up of wounds healing using area segmentation on a specific monoband plan.

\section{ACKNOWLEDGMENTS}

The authors would like to thank the financial support provided by Conseil Régional de Bourgogne, France, laboratoire Bioderma, France and Cancéropôle du Grand Est, France.

\section{REFERENCES}

1. S. Tominaga and Y. Moriuchi, "Principal component analysis-based reflectance analysis/synthesis of cosmetic foundation," Journal of Imaging Science and Technology 53, Issue 6, pp. 060403-(8), November/December 2009.

2. S. Prince and S. Malarvizhi, "Analysis of diffuse reflectance spectra of various skin conditions by principal component method," in Biomedical and Pharmaceutical Engineering, 2009. ICBPE '09. International Conference on, pp. $1-4$, dec. 2009.

3. H. Nugroho, M. Fadzil, V. Yap, S. Norashikin, and H. Suraiya, "Determination of skin repigmentation progression," in Engineering in Medicine and Biology Society, 200\%. EMBS 200\%. 29th Annual International Conference of the IEEE, pp. $3442-3445,2007$.

4. S. Cotton, "A noninvasive skin imaging system," tech. rep., Proceedings of the Conference on Intelligent Information Processing (IIP 2000) of the International Federation for Information Processing Sixteenth World Computer Congress (WCC 2000, 1997.

5. R. Jolivot, P. Vabres, and F. Marzani, "An open system to generate hyperspectral cubes for skin optical reflectance analysis," in Skin Research and Technology, 16 (4), (Besancon, France), November 2010. 
6. R. Jolivot, P. Vabres, and F. Marzani, "Reconstruction of hyperspectral cutaneous data from an artificial neural network-based multispectral imaging system," Computerized medical imaging and graphics $\mathbf{3 5}$ (2), pp. 85-88, March 2011.

7. A. Mansouri, F. Marzani, and P. Gouton, "Neural networks in two cascade algorithms for spectral reflectance reconstruction," in ICIP (2), pp. 718-721, 2005.

8. T. Fitzpatrick, "Soleil et peau," Journal de Medecine Esthetique 2, pp. 33-34, 1975.

9. J. Hernández-Andrés, J. Romero, and J. Raymond L. Lee, "Colorimetric and spectroradiometric characteristics of narrow-field-of-view clear skylight in granada, spain," J. Opt. Soc. Am. A 18(2), pp. 412-420, 2001. 